\title{
The Temperature Dependence of the Viscoelastic Behavior of Poly(vinyl acetate)
}

\author{
Donald J. PlazeK \\ Metallurgical and Materials Engineering, University of Pittsburgh, \\ Pittsburgh, PA 15261, U.S.A.
}

(Received September 6, 1979)

\begin{abstract}
The torsional creep and creep recovery behavior of a high molecular weight $\left(M_{w}=6.5 \times 10^{5}\right)$ poly(vinyl acetate) is reported for the temperature range, $37.5^{\circ} \mathrm{C}$ to $154^{\circ} \mathrm{C}$. The results for the recoverable compliance, $J_{\mathrm{r}}(t)$, reveal the usual two dispersions seen between glassy and steady state behavior. The intermediate rubbery plateau compliance, $J_{\mathrm{N}}$, has a value of $2.8 \times 10^{-7}$ $\mathrm{cm}^{2} \mathrm{dyn}^{-1}$, reflecting a molecular weight per entangled unit of 8,500 . Though the specimen studied was a fraction, the terminal dispersion was extensive. The steady-state recoverable compliance, $J_{\mathrm{e}}{ }^{\circ}$, was approximately 80 times larger than $J_{\mathrm{N}}$. Because there was considerable information on the terminal dispersion, it was possible to independently determine its temperature dependence, which was found to be indistinguishable from that of the viscous deformation. However, this temperature dependence is significantly different from that of the softening dispersion. The differences observed are in the same sense as that found previously for the softening dispersion and viscous flow dependences of polystyrene.
\end{abstract}

KEY WORDS Creep/Creep Recovery / Poly(vinyl acetate)/Viscoelasticity /

Temperature Dependence / Time-Temperature Superposition / Dynamic

Compliances / Dynamic Moduli /

It has been known for some time that the temperature dependence of the recoverable compliance, $J_{\mathrm{r}}(t)$, of polystyrenes with narrow molecular weight distributions is greater than that of the viscosity, at temperatures just above the glass temperature. This difference in temperature dependence is not specific for the polystyrene system, but is true also for other linear flexible chain amorphous polymers. Early work on polyisobutylene indicated a discrepancy between temperature dependences, ${ }^{4}$ but the reported differences were ignored, probably being ascribed primarily to experimental error. For similar reasons, we avoided reporting the results of certain viscosity measurements for a solution of cellulose nitrate. It was assumed that these results were incorrect since their variation with temperature did not agree with that determined from the viscoelastic response. A later study ${ }^{1}$ revealed discrepancies as large as an order or magnitude which could not be dismissed as simply error. Since the viscoelastic mechanisms with the longest relaxation times are the largest contributors to viscous deformations, it has been assumed that only the last few need have a different tempera- ture dependence to explain the failure of the reduction scheme for creep compliance curves which extend from the softening to the terminal zones. A recent analysis of some previously unpublished creep and creep recovery data on a linear poly(vinyl acetate) (PVAC), $\left(M_{w}=6.5 \times 10^{5}\right)$ reveals two temperature dependences with additional information on the terminal zone of the recoverable complaince.

\section{EXPERIMENTAL}

The creep, $J(t)$, and recoverable creep compliance, $J_{\mathrm{r}}(t)$ measurements were carried out at the Mellon Institute on a magnetic bearing torsional creep apparatus at eleven temperatures between $37^{\circ} \mathrm{C}$ and $155^{\circ} \mathrm{C}$. The specimens were measured in vacuum. The pressure, produced with just a mechanical vacuum pump, was about $10^{-2}$ Torr, which we have found is sufficiently low to remove absorbed moisture.

Absorbed moisture from the atmosphere was found to plasticize the poly(vinyl acetate) as it does most polymers. The effect is more marked for polar 
polymers but even polystyrene's creep velocity is markedly increased at temperatures near its glass temperature, $T_{\mathrm{g}}$, of $98^{\circ} \mathrm{C}$. Comparisons with the results of creep measurements made on PVAC exposed to the atmosphere ${ }^{5}$ showed that the velocity of creep was six times slower at temperatures near $38^{\circ} \mathrm{C}$ when the measurements were carried out in vacuum. Instrumental and procedural details are presented elsewhere. ${ }^{6}$

The principal sample studied was a fraction ${ }^{7}$ (5-72) kindly provided by Berry. It's weight average molecular weight, $M_{w},\left(6.5 \times 10^{5}\right)$ and other solution properties have been reported. ${ }^{8}$ The shear viscosity of the bulk polymer determined in this study is within $20 \%$ of the value determined and reported for $155^{\circ} \mathrm{C}$ by Berry et al. ${ }^{8}$ This check should allay any question concerning the possibility of degradation following the original dilute solution characterization and our later creep studies.

At the time these measurements were made, it was thought that variations in the stereoregularity of PVAC might be found as a consequence of differences in polymerization temperatures. Four fractions of samples with molecular weights close to $1 \times 10,{ }^{5}$ that had been polymerized either at $-70^{\circ} \mathrm{C}$ or $30^{\circ} \mathrm{C}$, were studied and the creep compliances in the softening dispersions were all found to be indistinguishable from each other and the results presented here. Other properties, including $\theta$ temperatures, of these and similar samples gave no indication of differences in tacticity. ${ }^{9}$

\section{RESULTS}

The recoverable shear creep compliance curves, $J_{\mathrm{r}}(t)$ measured at eleven temperatures from the neighborhood of the glass temperature, upward to $154^{\circ} \mathrm{C}$ are presented in Figure 1. At the three highest temperatures the total creep compliance curves, $J(t)$, including the viscous contributions are shown as dashed lines. The curves at the lower temperatures extend from the glassy compliance $\left(J_{\mathrm{g}}=0.90 \times 10^{-10}\right.$ $\mathrm{cm}^{2} \mathrm{dyn}^{-1}$ or $\left.10^{-9} \mathrm{~m}^{2} \mathrm{~N}^{-1}\right)$ up to an extensive rubbery plateau $\left(J_{\mathrm{N}} \cong 2.8 \times 10^{-7} \mathrm{~cm}^{2} \mathrm{dyn}^{-1}\right)$. The recoverable compliance, $J_{\mathrm{r}}(t)$, results obtained at temperatures above $75^{\circ} \mathrm{C}$ reveal a sizable terminal dispersion which rises to a steady state recoverable compliance, $J_{\mathrm{e}}^{\circ}, 80$ times larger than $J_{\mathrm{N}}$. This large terminal dispersion is unexpected since the specimen studied was a fraction with an estimated heterogeneity index, $M_{w} / M_{n}$, of 1.1. Most-high-molecularweight polymer samples with narrow molecular weight distributions show a molecular weight independent $J_{\mathrm{e}}^{\circ}$ which is about three times larger than

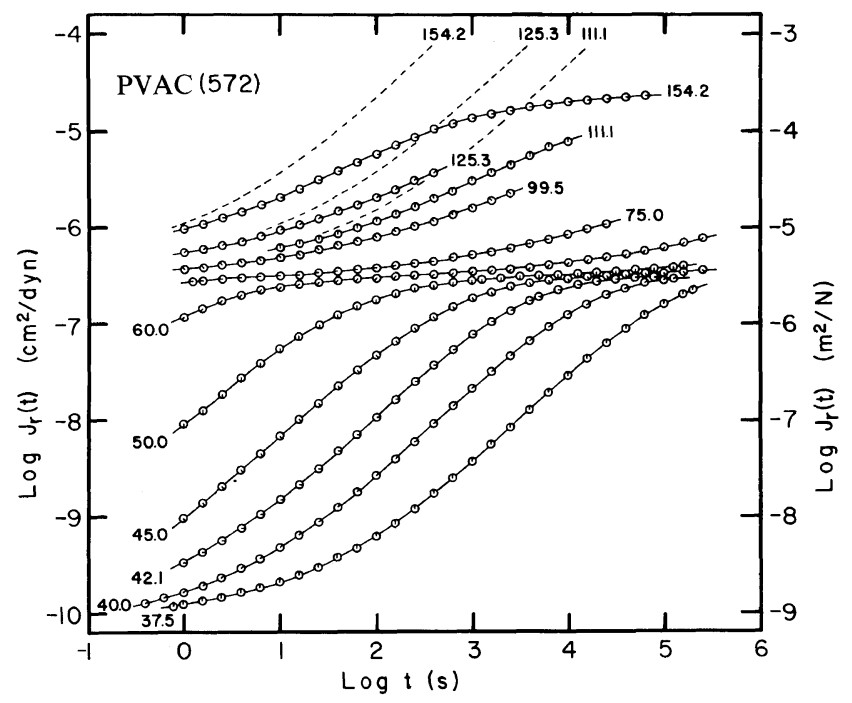

Figure 1. Logarithmic plot of the recoverable shear compliance, $J_{\mathrm{r}}(t), \mathrm{cm}^{2} \mathrm{dyn}^{-1}$, as a function of time (s), for a poly (vinyl acetate) sample $\left(M_{w}=6.5 \times 10^{5}\right)$ at eleven temperatures in ${ }^{\circ} \mathrm{C}$. Dashed lines indicate the total creep compliance, $J(t)$, determined at the three highest temperatures of measurement. 
$J_{\mathrm{N}}{ }^{3,10}$ Earlier measurements of similar PVAC fractions also displayed large terminal dispersions which tended to support the conclusion that the proportionality of $J_{\mathrm{e}}^{\circ}$ with molecular weight persisted at high molecular weights. ${ }^{5}$ We can only conclude that the propensity of PVAC, for free radical chain transferring, cantiot be suppressed sufficiently to eliminate long chain braching completely at high molecular weights. If this is so, the level of branching is so low as not to perceptibly affect dilute solution properties such as the intrinsic viscosity, whereas the recoverable compliance would have to reflect a great sensitivity to the presence of ranching.

However, in this study, the prominence of the terminal dispersion has contributed definitive information leading to the most significant conclusion of this report concerning the temperature dependences of the viscoelastic response. It has been possible to make an independent determination of the temperature dependence of the recoverable deformation accruing beyond the $J_{N}$ level. Figure 2 presents these contributions to the recoverable compliance as functions of the cube root of the time, $t$. The linear region of response is described as Andrade creep, ${ }^{11-13} J(t)=J_{\mathrm{A}}+\beta t^{1 / 3}$ where $J_{\mathrm{A}}$ and $\beta$ are characterizing constants. When Andrade creep is found near the glassy level of response, $J_{\mathrm{A}}$ is a valuable objective measure of the glassy compliance, $J_{\mathrm{g}}$, and when it is observed in the terminal dispersion, as it is here, $J_{\mathrm{A}}$ is identical to $J_{\mathrm{N}}$. Contributions of this form dominate only over a limited portion of the accessible time scale, but the values of $\beta$ obtained were used to calculate-time scale shift factors, $a_{\mathrm{T}}$, which are in excellent agreement with other values obtained from direct temperature reduction of recoverable compliance curves obtained which describe the terminal dispersion. That is, both sets of shift factors are fitted by the same equation.

$$
\log a_{\mathrm{T}}\left(J_{\mathrm{w}}, \beta\right)=-15.469+928 /(T+25)
$$

where $T$ is given in degrees Centigrade. This equation has the form of the Vogel, Tamman, Fulcher (VTF) equation,

$$
R=R_{0} \exp \frac{c}{T-T_{\infty}}
$$

where $R_{0}, C$, and $T_{\propto}$ are characteristic constants for a rate process of the material. In eq $1, T_{\propto}=-25^{\circ} \mathrm{C}$.

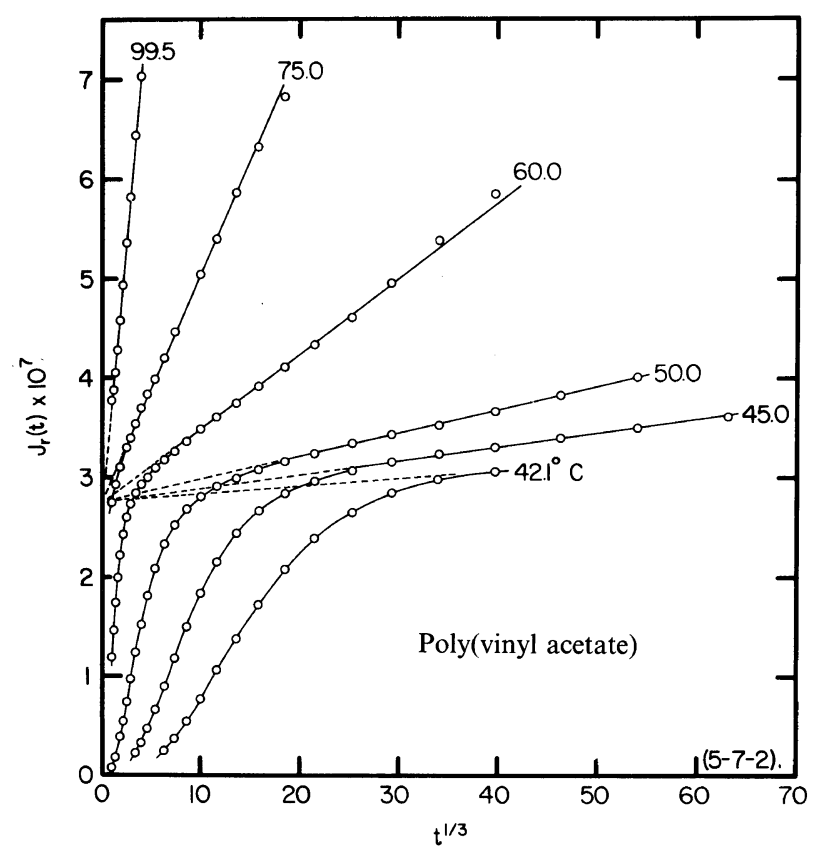

Figure 2. The recoverable compliance, $J_{\mathrm{r}}(t)$, plotted as a function of the cube root of time at 6 temperatures. Dashed lines are extrapolations of the Andrade creep region. 
We will identify data pertaining to the terminal dispersion with a subscript $w$, such as $J_{\mathrm{w}}$, and that pertaining to the softening dispersion with a subscript $\mathrm{s}$. The comparison made with shift factors calculated from simple viscosity ratios showed that the viscosity also has the same temperature dependence as described by eq 1 if it is recognized that,

$$
\log \frac{\eta\left(T^{\circ} \mathrm{C}\right)}{\eta\left(35^{\circ} \mathrm{C}\right)}=\log \eta\left(T^{\circ} \mathrm{C}\right)-17.05=\log a_{\mathrm{T}}(\eta)
$$

The shift factors obtained from shifting the recoverable compliance data from the softening dispersion, $J_{\mathrm{r}, \mathrm{s}}(t)$ are fitted with significantly different constants,

$$
\log a_{\mathrm{T}}\left(J_{\mathrm{s}}\right)=-12.80+373 /(T-6)
$$

where $T_{\propto}=+6^{\circ} \mathrm{C}$

The two temperature dependences are displayed in Figure 3 as solid lines, which show how the short time response mechanisms (those contributing to the softening dispersion) are more temperature sensitive near the glass temperature. All of the shift factors are presented in Table I.

\section{Temperature Reduction}

Since all of the viscoelastic mechanisms contribu- ting to the deformation of the poly(vinyl acetate) do not have the same temperature dependence, temperature reduction of the data to a single unique curve cannot be obtained. However, recognizing that if all of the mechanism contributing to a given dispersion have the same temperature dependence, successful reduction should be possible within the dispersion. We have therefore reduced the two dispersions seperately. Andrade plots were used to help assign data points to either the softening or the terminal dispersion. Points falling on or above each Andrade line in Figure 2 were assigned to the terminal dispersion. This procedure is an idealization and there is actually some ambiguity where the two groups of mechanisms overlap. The result of reduction of the $J_{\mathrm{r}}(t)$ data to $60^{\circ} \mathrm{C}$ is indicated by the data points in Figure 4. The dotted line indicates the total creep compliance, $J(t)$, which includes the viscous contributions. The dashed and dot-dashed lines indicate the results of reduction of the recovery and the creep, respectively, at a different temperature, $T_{0}=35^{\circ} \mathrm{C}$. The time scale has been shifted to superimpose the softening dispersion of the two reduced curves. The rubbery plateau is approximately 25 times shorter at the lower temperature because the softening dispersion has moved to long times more rapidly with the temperature change than

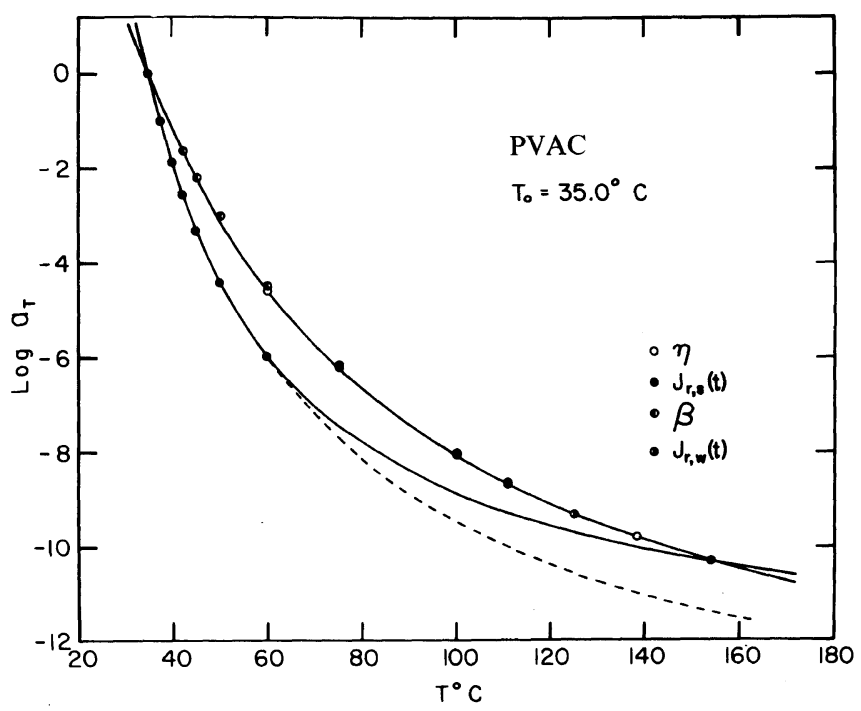

Figure 3. Logarithm of the temperature shift factors, $a_{\mathrm{T}}$, vs. the temperature: from softening dispersion, $J_{\mathrm{r}, \mathrm{s}}(t)$; $\bigcirc$, from the viscosity, $\eta$; $\boldsymbol{O}$, from the Andrade coefficients, $\beta$; $\boldsymbol{\top}$, from the terminal dispersion, $J_{\mathrm{r}, \mathrm{w}}(t)$. Dashed line from the compromise temperature dependence. The reference temperature, $T_{0},-35^{\circ} \mathrm{C}$. 
Poly(vinyl acetate) Creep Temperature Dependence

Table I. Temperature dependences

\begin{tabular}{|c|c|c|c|c|c|c|}
\hline$T /{ }^{\circ} \mathrm{C}$ & $\log \eta$ & $\log \beta$ & $\log a_{\mathrm{T}}\left(J_{\mathrm{w}}\right)$ & $\log a_{\mathrm{T}}(\eta)$ & $\log a_{\mathrm{T}}(\beta)$ & $\log a_{\mathrm{T}}\left(J_{\mathrm{s}}\right)$ \\
\hline 35.0 & $17.05^{\mathrm{a}}$ & $-9.64^{a}$ & 0.00 & 0.00 & 0.00 & 0.00 \\
\hline 37.5 & & & & & & -1.03 \\
\hline 40.0 & & & & & & -1.87 \\
\hline 42.1 & & -9.09 & & & -1.64 & -2.56 \\
\hline 45.0 & & -8.94 & & & -2.10 & -3.31 \\
\hline 50.0 & & -8.65 & & & -2.99 & -4.41 \\
\hline 60.0 & 12.47 & -8.15 & & -4.58 & -4.48 & -5.95 \\
\hline 75.0 & 10.83 & -7.59 & -6.19 & -6.22 & -6.14 & \\
\hline 99.5 & 9.00 & -6.95 & -8.01 & -8.05 & -8.06 & \\
\hline 111.1 & 8.38 & & -8.65 & -8.68 & & \\
\hline 125.3 & 7.75 & & -9.29 & -9.31 & & \\
\hline 138.2 & 7.25 & & & -9.80 & & \\
\hline 154.2 & 6.76 & & -10.29 & -10.29 & & \\
\hline
\end{tabular}

${ }^{a}$ Calculated.

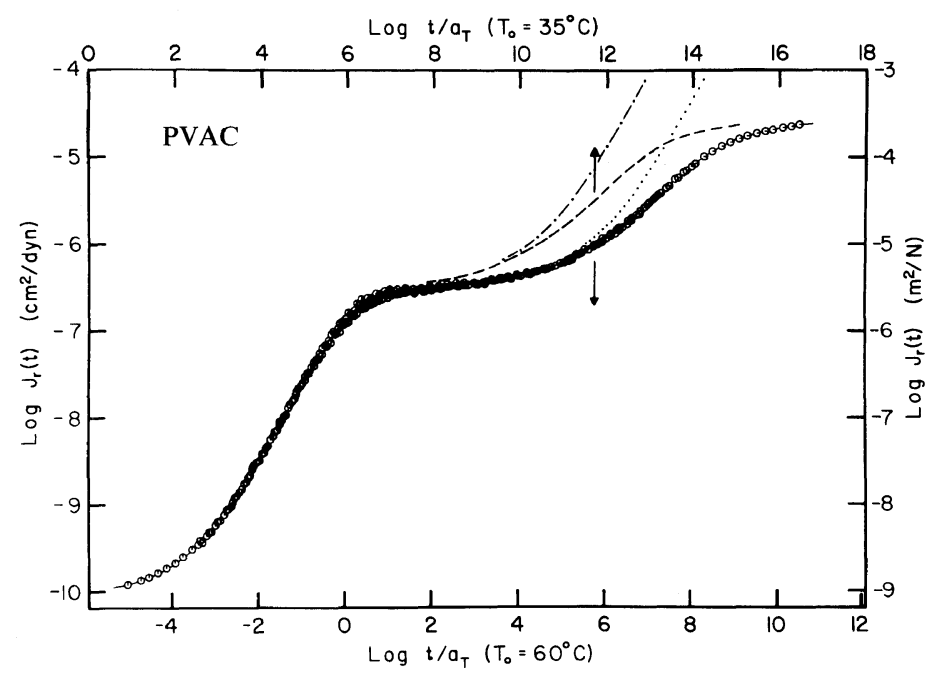

Figure 4. Logarithmic plot of $J_{\mathrm{r}}(t) v s$. the reduced time scales, $t / a_{\mathrm{T}}$, with reference temperatures of $60^{\circ} \mathrm{C}$ (data points) and $35^{\circ} \mathrm{C}$ (dashed line). Corresponding $J(t)$ are curves dotted and dash-dotted lines, respectively.

has the terminal dispersion. The lack of complete superposition at the beginning of the rubbery plateau is the result of the ambiguity in the overlap region.

If one attempts tỏ obtain a reduced curve of all the data (ignoring the two distinct temperature dependences) with one compromise temperature dependence, a curve is obtained for $T_{0}=35^{\circ} \mathrm{C}$ that is coincidentally indistinguishable in shape from that obtained at $60^{\circ} \mathrm{C}$ using the two temperature de- pendences. The compromise equation used is as follows.

$$
\log a_{\mathrm{T}}=-15.14+596 /(T+5)
$$

where $T_{\infty}=-5^{\circ} \mathrm{C}$. It is shown as a dashed line in Figure 3.

The best fits of the data to the VTF equation, which is equivalent to the William, Landel and Ferry (WLF) free volume equation, are seen in Figure 3 to surprisingly cross at high temperatures $\left(\sim 154^{\circ} \mathrm{C}\right)$. If 
this actually were the case, reduction of the data to temperatures higher than $60^{\circ} \mathrm{C}$ would show a reversal in the rubbery plateau length; i.e., an increase in temperature would lead to a shortening of the plateau. This would be in the direction observed in the behavior of some of the polymethacrylates, ${ }^{14}$ which has been interpreted as a change in the entanglement spacing. It is also possible that the reduced curve obtained at $60^{\circ} \mathrm{C}$ could represent an asymptotic high temperature form which will persist at all higher temperatures. In this latter case, the compromise temperature dependence equation, eq 5 , would coincidentally represent more realistically the actual behavior to be found at higher temperatures for the softening dispersion; see the dashed line in Figure 3. Measurements would have to be made at moderately high frequencies or at much shorter times in the softening dispersion should be able to distinguish between the alternative possibilities. Since the WLF and the VTF equations are equivalent temperature forms, which implicitly reflect the changes of the specific volume, ${ }^{15}$ and since their strict applicability may be limited, it is a distinct possibility that what is basically the Doolittle free volume form,

$$
R=R_{0} \mathrm{e}^{b / \phi_{f}}
$$

(where $R_{0}$ and $b$ are characterizing constants and $\phi_{\mathrm{f}}$ is the fractional free volume; $\phi=\left(v-v_{0}\right) / v_{0}, v$ is the measured volume and $v_{0}$ is the occupied volume) does not accurately hold even within $100^{\circ} \mathrm{C}$ above $T_{\mathrm{g}}$ for the softening dispersion.

\section{Retardation Spectrum}

The distribution functions of retardation times, $\tau$, i.e., the retardation spectra, $L(\ln \tau)$, corresponding to the two reduced recoverable compliance curves displayed in Figure 4 were determined empirically with iterative variations until each $J_{\mathrm{r}}(t)$ curve could be calculated from the spectra within $\pm 2 \%$ for all points. Numerical integration on a computer of the expression,

$$
J_{\mathrm{r}}(t) \equiv J(t)-t / \eta=J_{\mathrm{g}}+\int_{-\infty}^{+\infty} L\left(1-\mathrm{e}^{t / \tau}\right) \mathrm{d} \ln \tau
$$

was used to ascertain the quality of the proposed spectrum, $L$. The results of the estimations are shown in Figure 5, where the principal features usually exhibited by high molecular weight amorphous polymers are seen: 1) a slope on a $\log -\log$ plot of $L v s$.

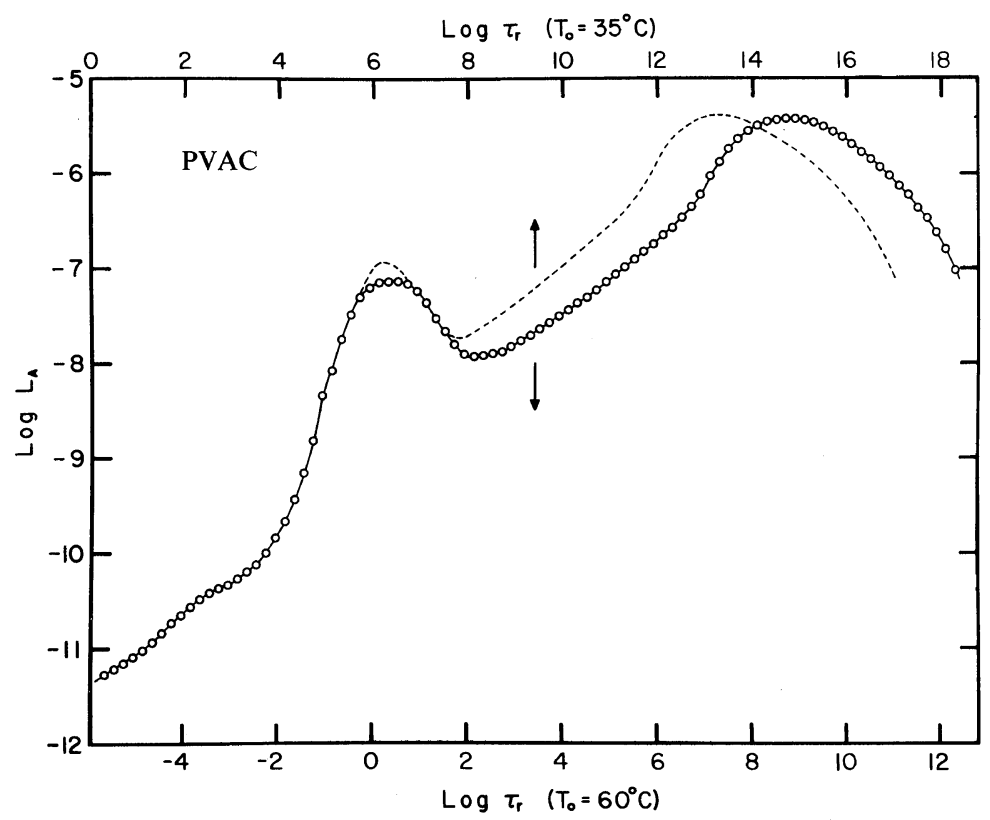

Figure 5. Logarithmic plot of the acceptable retardation spectra, $L_{\mathrm{A}}$, vs. the logarithm of the reduced retardation times, $\tau_{\mathrm{r}}$. The line with points was determined for the $J_{\mathrm{r}}(t)$ curve reduced to $60^{\circ} \mathrm{C}$ in Figure 4 and the dashed line for the $35^{\circ} \mathrm{C}$ curve. 
the reduced retardation time, $\tau_{\mathrm{r}}$, of $1 / 3$ at short times in the glassy region of response, indicating the presence of Andrade creep, (We take the slight shoulder at $\log \tau_{\mathrm{r}}\left(T_{0}=60^{\circ} \mathrm{C}\right)=-4$ to be caused by experimental uncertainty since the $\beta$ dispersion is known to be far removed ${ }^{16}$ ); 2) a sizable symmetrical peak reflecting retarded cooperative motions of polymer chain segments between entanglements, (The differences at the maximum are the result of the ambiguity in the curves in the region of mechanism overlap referred to above); 3) a second Andrade creep region dominating the deformation in the rubbery plateau region, which is the beginning of the terminal dispersion, reflects the adjustments of the entanglement network.

In these cases, the approach to the terminal peak involves rising above the nearly linear segment with the $1 / 3$ slope. This is a feature we have not seen before and is presumably due to the form of the molecular weight distribution or the presence of long-chain branching. The most objective measure for the length of the rubbery plateau, we believe, is the separation of the two peaks of $L$. The shorter plateau for the curve reduced to $35^{\circ} \mathrm{C}$ is clearly seen.

\section{Dynamic Mechanical Properties}

Using the spectrum, reduced to $60^{\circ} \mathrm{C}$, the various dynamic viscoelastic functions were calculated on a computer with numerical integration programs.* The components of the complex dynamic shear compliance,

$$
J^{*}(\omega)=J^{\prime}(\omega)-i J^{\prime \prime}(\omega)
$$

where $J^{\prime}(\omega)$, the real component, is the storage compliance and $J^{\prime \prime}(\omega)$, the imaginary component, is the loss compliance, were calculated using the

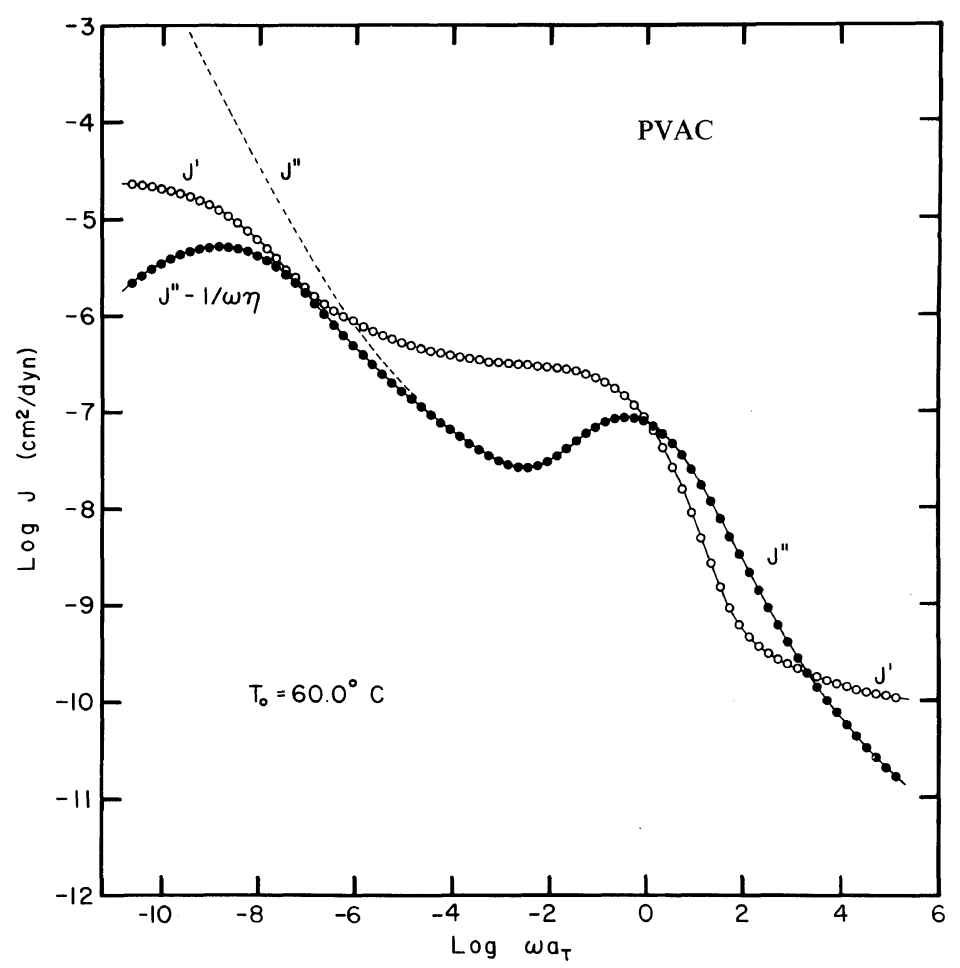

Figure 6. Double logarithmic plots of the dynamic compliances as a function of the reduced frequency scale, $\omega a_{\mathrm{T}}$. Calculated from the $L$ reduced to $60^{\circ} \mathrm{C}$.

* These programs and those used to determine the retardation spectra which yield our reduced recoverable compliance curves were written by Dr. Stephen J. Orbon. 
expressions,

$$
J^{\prime}(\omega)=J_{g}+\int_{-\infty}^{\infty} \frac{L}{1+\omega^{2} \tau^{2}} \mathrm{~d} \ln \tau
$$

and

$$
J^{\prime \prime}(\omega)=\int_{-\infty}^{\infty} \frac{\omega \tau}{1+\omega^{2} \tau^{2}} \operatorname{dln} \tau+\frac{1}{\omega \eta}
$$

The results are plotted in Figure 6. The loss compliance, without its simple viscous contribution, $J^{\prime \prime}-1 / \omega \eta$, which is the zeroth approximation to $L$ where $\tau^{-1}=\omega$ the angular frequency in $\operatorname{rad~s}^{-1}$, is emphasized at low frequencies since the less informative $1 / \omega \eta$ contribution dominates $J^{\prime \prime}(\omega)$ at $\omega$ $<10^{-8} \mathrm{~s}^{-1}$. The glassy, rubbery, and steady state plateaus are clearly visible in the $\log J^{\prime}$ curve. Each dispersion in $J^{\prime}$ is associated with a corresponding maximum in $J^{\prime \prime}-1 / \omega \eta$.

The components of the complex dynamic shear modulus

$$
G^{*}(\omega)=G^{\prime}(\omega)+i G^{\prime \prime}(\omega)
$$

where $G^{\prime}(\omega)$ is the storage modulus and $G^{\prime \prime}(\omega)$ is the loss modulus were calculated from $J^{\prime}(\omega)$ and $J^{\prime \prime}(\omega)^{17}$ and plotted in Figure 7. The real component of $G^{*}(\omega)$ is $G^{\prime}(\omega)$, which drops from a glassy modulus very close to $10^{10} \mathrm{dyn} \mathrm{cm}^{-2}$ at high reduced frequencies down to the rubbery plateau with decreasing frequency. It finally decreases at a limiting rate proportional to $\omega^{2}$ or with a slope of 2 on the log-log plot at

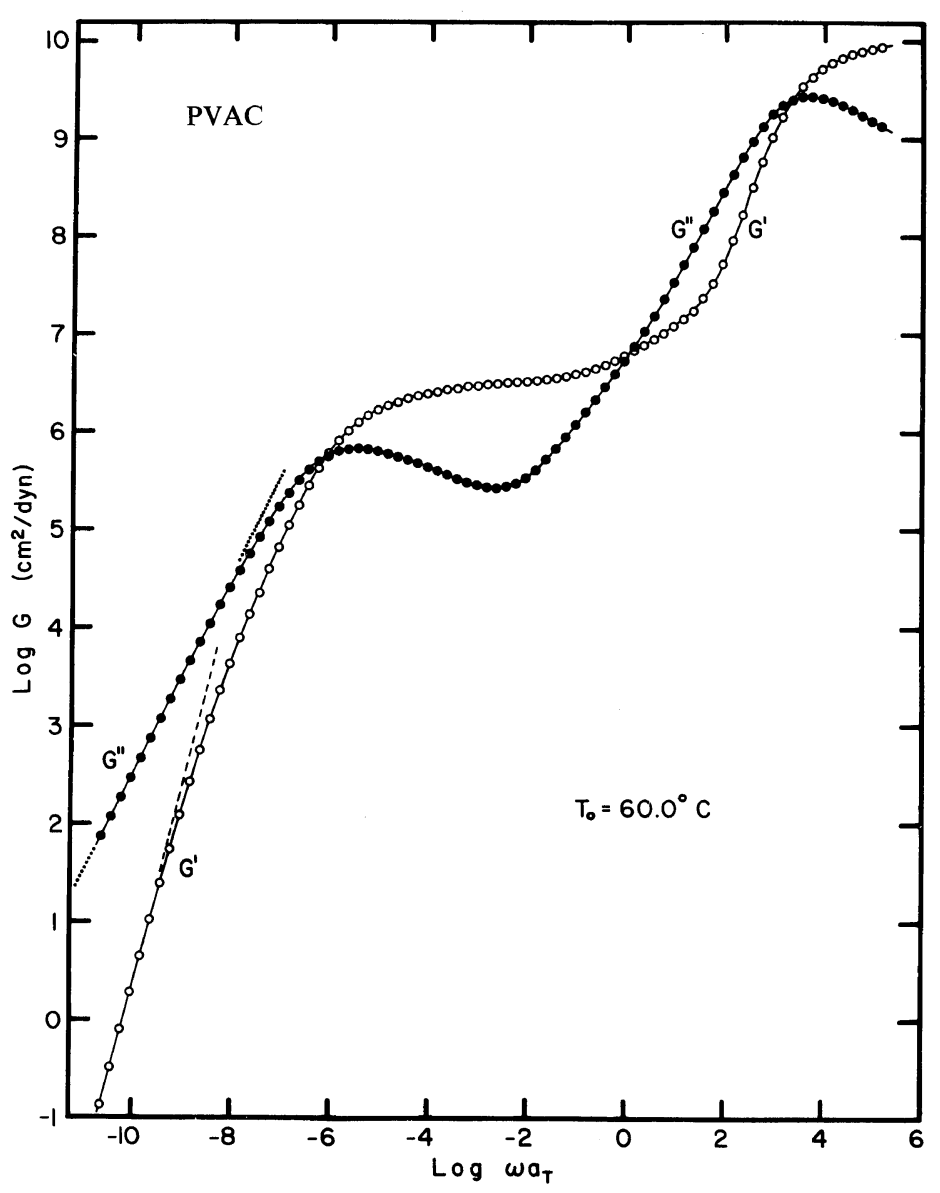

Figure 7. Double logarithmic plots of the storage, $G^{\prime}$, and loss, $G^{\prime \prime}$, dynamic moduli as functions of the reduced frequency scale. Calculated from the $L$ reduced to $60^{\circ} \mathrm{C}$. Dotted and dashed lines have the limiting slopes of one and two respectively. 
the lowest frequencies. After exhibiting two maxima at the higher frequencies, the loss modulus, $G^{\prime \prime}(\omega)$, follows $G^{\prime}(\omega)$ toward zero, diminishing at a rate directly proportional to $\omega$ or with a slope of one on the log-log plot. These limiting slopes are simply consequences of the fact that the PVAC studied is not in a viscoelastic liquid. In the limiting region, the asymptotic values of $G^{\prime \prime}(\omega) / \omega=\eta_{0}$ where $\eta_{0}$ is the limiting low rate of shear viscosity and $G^{\prime}(\omega)$ / $\omega^{2}=\eta^{2} J_{\mathrm{e}}$.

Finally, the real and imaginary components, $\eta^{\prime}(\omega)$ and $\eta^{\prime \prime}(\omega)$, of the complex dynamic viscosity,

$$
\eta^{*}(\omega)=\eta^{\prime}(\omega)-i \eta^{\prime \prime}(\omega)
$$

where calculated using the relations $G^{\prime}=\omega \eta^{\prime}$ and $G^{\prime \prime}=\omega \eta^{\prime} ; G^{*}=i \cdot \omega \eta^{*}$. These curves are not often seen and a few of their features are not commonly appreciated.

$$
\left|\eta^{*}\right|=\left(\eta^{\prime 2}+\eta^{\prime 2}\right)^{1 / 2}
$$

is so close to the larger of the components that it is barely perceptible as a separate curve. It can be seen as a dotted line in Figure 8 as it switches from $\eta^{\prime}$ to $\eta^{\prime \prime}$ and back again. It is also clear that $\eta^{\prime}$ and $\eta^{*}$ decrease from the low frequency asymptote, $\eta_{0}$, with increasing frequency to a plateau in the neighborhood of $\log \omega a_{\mathrm{T}}=0$ after which both resume decreasing at higher frequencies. Meanwhile, $\eta^{\prime \prime}(\omega)$ passes through two maxima as do the other imaginary components.

\section{DISCUSSION}

The behavior of poly(vinyl acetate) fractions have been perplexing and misleading. In addition to noting that the magnitude of the terminal dispersion is not typical of polymers whose molecular weight distribution has been known to be narrow, we can point to the length of the rubber-like plateau which is longer than expected for a narrow distribution polymer. In making this statement, we are assuming that the behavior exhibited by "monodisperse" polystyrene ${ }^{18-20}$ is expected for all linear amorphous polymers with flexible molecules. It has been established that the rubbery plateau length measured by the separation of the peaks in $L$ is

$$
\Delta \log \tau_{\max }=3,4 \log \left(M / M_{\mathrm{c}}\right)
$$

when $M$ is the molecular weight and $M_{\mathrm{c}}$ is the critical value of the molecular weight at which molecular entanglements start influencing the viscosity; i.e., the molecular weight at which the break in the $\log \eta v s$. $\log M_{w}$ curve occurs. No molecular weight moments are indicated in eq 14 because it underestimates the width of the plateau for broad distribution polymers. ${ }^{21}$ From our experience with the PVAC, data we judge that the earlier polystyrene analysis most likely represents the high temperature asymptotic lengths of the rubbery plateaus because what were obviously single compromise temperature depend-

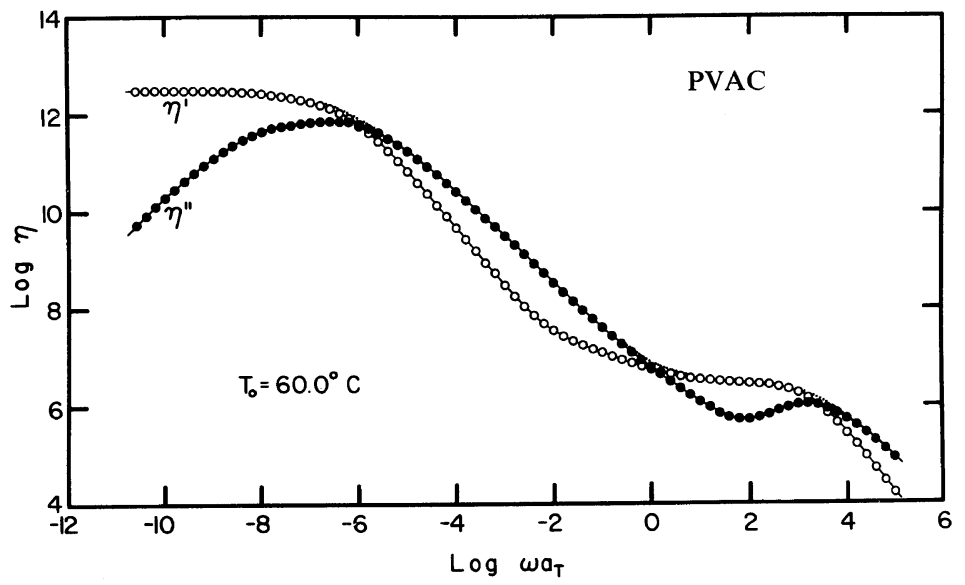

Figure 8. Real, $\eta$, and imaginary, $\eta^{\prime \prime}$, components of the complex dynamic shear viscosity plotted logarithmically as a function of the logarithmic reduced frequency scale. Calculated from the modulus curves of Figure 7. The dotted line represents the absolute value of $\eta^{*}$. 
ence equations were used in the reduction process. The separation of the maxima in the $60^{\circ} \mathrm{C}$ retardation spectrum is $\Delta \log \tau_{\max }=8.6$ and $\log M_{w}=5.81$. These values yield an " $M_{\mathrm{c}}$ " $=1,900$. If we use the $35^{\circ} \mathrm{C}$ spectrum, where $\Delta \log \tau_{\max }=7.2$ we obtain " $M_{\mathrm{c}}$ " $=4,900$. In reality $M_{\mathrm{c}}$ for PVAC is $24,500^{22}$. Therefore, based on the molecular weight dependence of the viscoelastic response of polystyrene, we conclude that the rubbery plateau observed for this PVAC sample is much too wide for its molecular weight. We are not convinced that the presence of a slight amount of long chain branching is the reason why PVAC fractions behave as if they are not at all narrow in distribution but we do believe that the effect is chronic with the fractions we have studied.

The molecular weight between entanglements is calculated from the rubbery plateau compliance, $J_{\mathrm{N}}$, using the simple rubberlike expression,

$$
J_{\mathrm{N}}=M_{\mathrm{e}} /(\rho R T)
$$

However, we have three slightly different values for $J_{\mathrm{N}}=2.8 \times 10^{-7}, 3.2 \times 10^{-7}$ and $3.4 \times 10^{-7}$. The first is the average Andrade intercept and the latter two are the results of integrations under the first peak in $L$ as a function of the natural logarithm of the retardation times of the $60^{\circ} \mathrm{C}$ and the $35^{\circ} \mathrm{C} L$ 's, respectively. Since the reduced curves involve distortions related to the above mentioned ambiguities, we believe the Andrade intercept to be the most reliable. The resulting $M_{\mathrm{e}}$ is 8500 . The ratio $M_{\mathrm{c}} / M_{\mathrm{e}}$ for poly(vinyl acetate) is therefore 2.9 , which is higher than the expected value of two. ${ }^{23}$ Since a slight amount of long-chain branching does not effect the value for $J_{\mathrm{N}}$, it is suggested that the currently accepted value for $M_{\mathrm{c}}$ could be high because of the suspected branching.

In regard to the two temperature dependences being reported here, one can note that it seems safe to assume in general that groups of viscoelastic mechanisms contributing to different maxima in $\left[J^{\prime \prime}(\omega)-1 /(\omega \eta)\right]$ or $G^{\prime \prime}(\omega)$ will a priori have different temperature dependences. The high temperature cross-over of the two temperature dependence curves as seen in Figure 3 is not unique to PVAC. Reexamining the earlier reported polystyrene data, we found that resulting equations also lead to the same kind of high temperature cross-over not noticed before.

Finally, we wish to point out that it is reassuring that the terminal dispersion of $J_{\mathbf{r}}(t)$ has the same temperature as that of the viscosity since they are both determined by the disentangling of the entanglement network. The contributions to $J_{\mathrm{r}}(t)$ above $J_{\mathrm{N}}$ are seen somewhat in advance of measurable contributions of the viscous deformation to $J(t)$ but this $t / \eta$ contribution to $J(t)$ is always dominant before $J_{\mathrm{e}}$ is reached.

Acknowledgements. The measurements reported here were made with the support of the National Aeronautics and Space Administration under Research Grant NsG 147-61. The author wishes to thank V. Michael O'Rourke for his help with the measurements. The data analysis was carried out under the principal support of the National Science Foundation, under Engineering Division Grant Eng-7818838. Thanks are also due to Linda Berardelli for assisting with the data analysis and figure preparation.

\section{REFERENCES}

1. D. J. Plazek, J. Phys. Chem., 69, 3480 (1965).

2. D. J. Plazek anv V. M. O'Rourke, J. Polym. Sci., A-2, 9, 209 (1971).

3. E. Riande, H. Markovitz, D. J. Plazek, and N. Raghupathi, J. Polym. Sci., Polym. Symp. No. 50, 405 (1975).

4. J. D. Ferry, L. D. Grandine, and E. R. Fitzgerald, $J$. Appl. Phys., 24, 911 (1953).

5. K. Ninomiya and J. D. Ferry, J. Phys. Chem., 67, 2292 (1963).

6. D. J. Plazek, J. Polym. Sci., A-2, 6, 621 (1968).

7. V. C. Long, G. C. Berry, and L. M. Hobbs, Polymer, 5, 517 (1964).

8. G. C. Berry, L. M. Hobbs, and V. C. Long, Polymer, 5, 31 (1964).

9. T. G Fox, unpublished results.

10. W. W. Graessley, Adv. Polym. Sci., 16, 1 (1974).

11. E. N. da C. Andrade, Proc. R. SQc. London, Ser. A, 84, 1 (1910); Phil. Mag., 7, 2003 (1962).

12. D. J. Plazek, W. Dannhauser, and J. D. Ferry, J. Colloid Sci., 16, 101 (1961).

13. D. R. Reid, Br. Plast. (October 1959).

14. J. D. Ferry, " Viscoelastic Properties of Polymers," 2nd ed, Wiley, New York, N.Y., 1970, Chapter 11, Section F2.

15. M. L. Williams, R. F. Landel, and D. J. Ferry, J. Am. Chem. Soc., 77, 3701 (1955).

16. N. G. McCrum, B. E. Read, and G. Williams, "Anelastic and Dielectric Effects in Polymeric Solids," Wiley, London, 1967, p 305.

17. J. D. Ferry, "Viscoelastic Properties of Polymer's," 2nd, Wiley, New York, N. Y., 1970, Chapter 1, 


\section{Poly(vinyl acetate) Creep Temperature Dependence}

Section C4.

18. D. J. Plazek, Am. Chem. Soc. Coat. and Plast. Prepr., 35, 389 (1975).

19. N. Raghupathi, Ph.D. Thesis, University of Pittsburgh, 1975.

20. S. J. Orbon and D. J. Plazek, J. Polym. Sci., in press.

21. D. J. Plazek and P. K. Agarwal, Proceedings, VII
International Congress on Rheology, August 1976, p 488 .

22. G. C. Berry and T. G Fox, Adv. Polym. Sci., 5, 261 (1967).

23. J. D. Ferry, "Viscoelastic Properties of Polymers," 2nd, Wiley, New York, N.Y., 1970, Chapter 10, Section C1. 